\title{
The Influence of Left Ventricular Dysfunction on The Early and Midterm Outcome in The Patients Undergoing Off Pump Coronary Artery Bypass Grafting Assessed by Propensity Matched Score Analysis
}

\author{
Tomonori Shirasaka, ${ }^{1,2}$ Nuttapon Arayawudhikul, ${ }^{2}$ Apichat Tantraworasin,,${ }^{3,4}$ Angsu Chartrungsan, ${ }^{2}$ \\ Boonsap Sakboon, ${ }^{2}$ Jaroen Cheewinmethasiri, ${ }^{2}$ Hiroyuki Kamiya ${ }^{1}$ \\ ${ }^{1}$ Division of Cardiothoracic Surgery, Lampang Hospital, Lampang, Thailand; \\ ${ }^{2}$ Division of Cardiac Surgery, Asahikawa Medical University Hospital, Hokkaido, Japan; \\ ${ }^{3}$ Department of Surgery, Faculty of Medicine, Chiang Mai University, Chiang Mai, Thailand; \\ ${ }^{4}$ Clinical Epidemiology and Clinical Statistic Unit, Faculty of Medicine, and Pharmacoepidemiology and Statistics Research Center \\ (PESRC), Faculty of Pharmacy, Chiang Mai University, Chiang Mai, Thailand
}

\section{ABSTRACT}

Background: It still remains unclear the depth of influence of left ventricular dysfunction on the recovery of patients' physical conditions in the early and midterm period following off-pump coronary artery bypass grafting (OPCAB).

Methods: From April 2011 to May 2018, 851 patients underwent $\mathrm{OPCAB}$ in our center. All were grouped into two groups: Those whose ejection fraction $(\mathrm{EF})$ was under $35 \%$ were defined as the Low EF group $(N=158)$ and those who maintained $\mathrm{EF}$ over $35 \%$ were defined as the Faired EF group $(N=693)$. Preoperatively, there was significant difference in NYHA class $(P<0.001)$, CCS class $(P=0.038)$, level of creatinine $(P<0.001)$, and rate of establishment of IABP $(P<0.001)$.

Results: Regarding all-cause death in the early postoperative period, low $\mathrm{EF}$ was a not a risk factor in patients $(P=0.52)$ or in the matched cohort $(P=0.398)$; however, in the midterm, it was a significant risk factor in patients (HR 2.07, $P=$ $0.016)$ and in the matched cohort (HR 2.72, $\mathrm{P}=0.029)$. Overall survival at 5 years in the Low EF group was significantly inferior to that of the Faired EF group in all $(67.4 \pm 4.1 \%$ and $86.1 \pm 2.9 \%, P=0.001)$ and in the matched cohort $(66.5 \pm 6.4 \%$ vs. $86.5 \pm 4.5 \%, P=0.008)$.

Conclusion: $\mathrm{OPCAB}$ seems beneficial for patients with LV dysfunction considering the early outcome, however, low $\mathrm{EF}$ is a significant risk factor for overall death in the midterm period.
Received September 18, 2021; accepted October 18, 2021.

Correspondence: Tomonori Shirasaka, Department of Cardiac Surgery, Asabikawa Medical University, Midorigaoka Higashi 2-1-1-1, Asabikawa 0788510, Japan, Telephone 81-166-68-2494 (e-mail: shira.skyrocket@gmail.com).

\section{INTRODUCTION}

Historically, left ventricular (LV) dysfunction has been regarded as a strong influencer for operative mortality [Christakis 1989; Kuroda 2016; Appoo 2004; Topkara 2005; Pieri 2016] because the usage of cardiopulmonary bypass and period of arrested heart may lead to the deterioration of the recovery of LV function in the early phase of the postoperative period. Previous studies have reported operative mortality rate after coronary artery bypass grafting (CABG) for those with LV dysfunction reached around 5\% [Pieri 2016]. Offpump CABG seems more beneficial to those patients because this mode of surgery guarantees the pulsatile circulation for every organ all through the operation and hence, contribute to early recovery of systemic condition [Kuss 2010; Marui 2012]. In addition, with the skill of an experienced surgeon and surgical team, this technique should be justified [Taggart 2016] although beating heart surgery for patients with LV dysfunction may drive a higher chance of emergence of arrhythmia or the difficulty in handling the enlarged LV.

In reality, there still is no established consensus for the superiority of OPCAB for this cohort to conventional onpump arrested CABG. Keeling et al. reported with metaanalysis that $\mathrm{OPCAB}$ contributed the significant decrease of early mortality compared with conventional CABG (Inhospital mortality assessed by volume-stratified risk-adjusted outcome: OR 0.44, $P=0.01$ ) [Keeling 2013]. Fukui et al. also concluded from their analysis with 161 patients with low EF who underwent $\mathrm{OPCAB}$ that early postoperative and follow-up patency rates of anastomoses were favorable $(98.3 \%$ and $85.8 \%$, respectively) and echocardiographic recovery of EF was also satisfactory [Fukui 2014]. In contrast to these reports, additional research claimed that an outcome comparison between $\mathrm{OPCAB}$ and conventional CABG did not show a significant difference in early mortality [Shroyer 2009; Møller 2008].

Our division has an established strategy with performing $\mathrm{OPCAB}$ in routine fashion, expecting the early recovery of the patient's condition. The aim of this study was to reveal the feasibility of OPCAB for patients with low EF on a regular 
basis and assess the early and midterm clinical outcomes compared with those with good or faired EF.

\section{PATIENTS AND METHODS}

Patient characteristics: From April 2011 to May 2018, 907 people underwent CABG in our center. Of all, 851 patients $(93.8 \%)$ underwent OPCAB and 56 patients $(6.2 \%)$ who had been very sick with multiple small, diffused target vessels and required mechanical support with multi-inotropic drugs simultaneously underwent on-pump arrested CABG (ONCAB). In this study, the former retrospectively was reviewed from medical records.

Patients were grouped into two groups: Those whose ejection fraction (EF) was under $35 \%$ were defined as the Low EF group $(N=158)$ and those who maintained EF over $35 \%$ were defined as the Faired EF group $(N=693)$.

Preoperative patient profile of the Low EF and the Faired EF groups are shown in Table 1. (Table 1) The subjects were
478 males and 373 females. A mean age of the two groups were not significantly different (the Low EF vs. Faired EF groups, $64.9 \pm 9.4$ vs. $64.3 \pm 8.3$ years, $P=0.393)$. New York Heart Association classification (NYHA class) and Canadian Cardiovascular Society classification (CCS class) were significantly different between the two groups (NYHA class, $3.3 \pm 0.6$ vs. $2.6 \pm 0.6, P<0.001$; CCS class, $3.3 \pm 0.5$ vs. $3.2 \pm 0.5$, $P=0.038$, Table 1$)$.

In relation to comorbid disease, chronic obstructive pulmonary disease (COPD), peripheral vascular disease (PVD), recent myocardial infarction (recent $\mathrm{MI}$ ), and renal insufficiency defined as an estimated glomerular filtration rate [Matsuo 2009] (GFR) less than $60 \mathrm{~mL} \cdot$ Min-1 $\cdot 1.73 \mathrm{~m}-2$ were different significantly (COPD, 16.5 vs. $10.3 \%, P=$ 0.036; PVD, 18.4 vs. $11.7 \%, P=0.035$; recent $M I, 77.2$ vs. $60.0 \%, P<0.001$; renal insufficiency, 28.5 vs. $15.7 \%, P<$ 0.001 , Table 1). The percentage of patients who required preoperative intra-aortic balloon pumping (IABP) support between the two groups were $38.6 \%$ and $14.1 \%$, respectively $(P<0.001$, Table 1$)$. The average number of coronary artery

Table 1. Patient characteristics in all cohort and the matched cohort

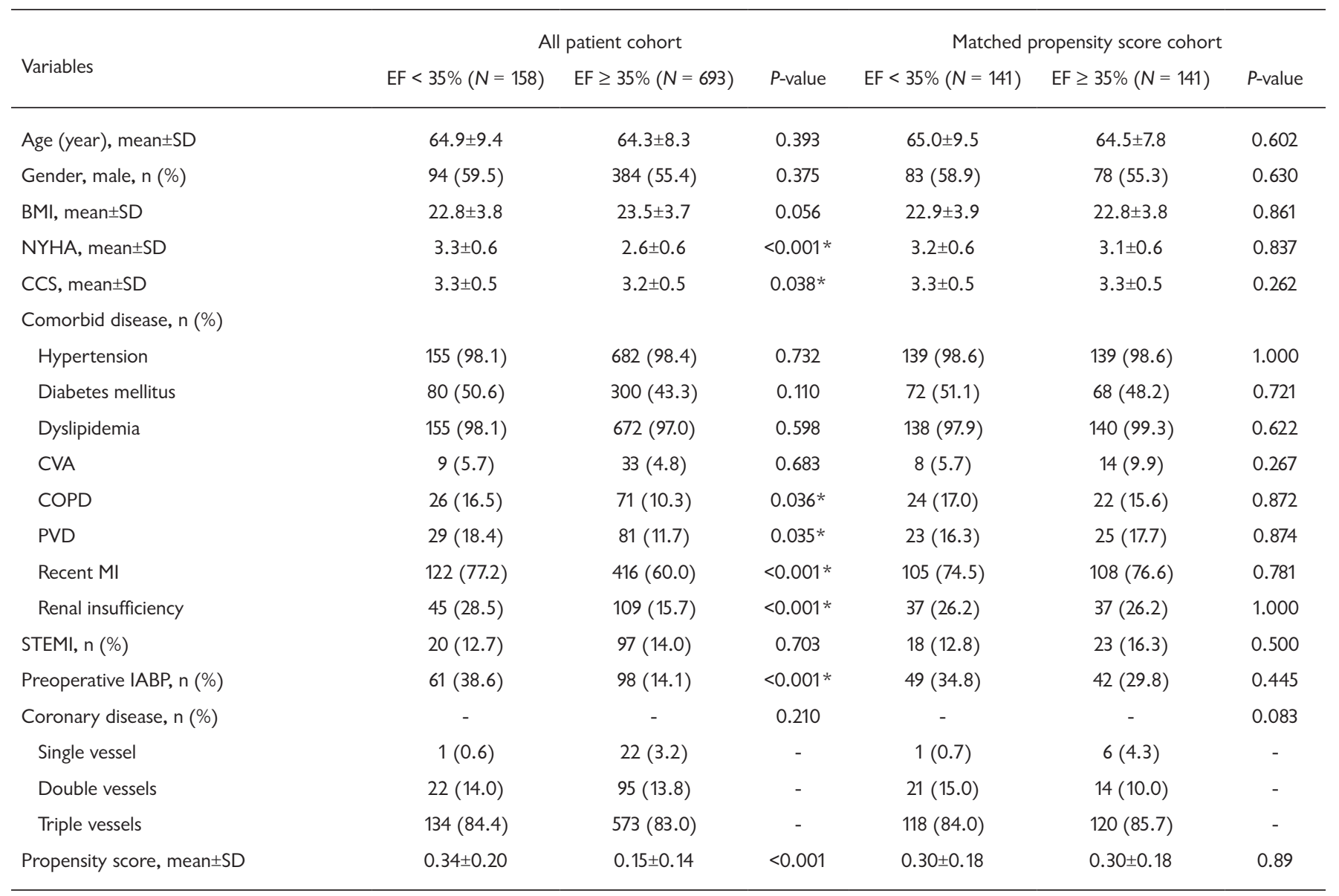

BMI, body mass index; NYHA, New York Heart Association; CCS, Canadian Cardiovascular Society; CVA, cerebrovascular accident; COPD, chronic obstructive pulmonary disease; PVD, peripheral vascular disease; MI, myocardial infarction; IABP, intra-aortic balloon pump; STD, standardized differences (STD of 0.2 or more indicates that covariates are imbalanced between groups) 
diseases between the Low EF and Faired EF group were not statistically different $(P=0.210$, Table 1$)$.

The hard-end $\neg$ points were overall death and any major adverse cardiac or cerebrovascular event (MACCE). MACCE included myocardial infarction, cardiac death, heart failure requiring hospitalization, repeat revascu $\neg$ larization, and stroke.

The institutional review board of Lampang Hospital approved this retrospective study and waived the need for written patient consent.

Surgical procedure: In this study, all patients were revascularized in off-pump fashion. Operative procedures were as follows: A median sternotomy was performed and fol-lowed by harvesting of the internal mammary artery in full skeletonized fashion. The target vessel for each anastomosis was appropriately exposed using tissue stabilizer (Octopus tissue stabilizer®. Medtronic Inc., Minneapolis, Minnesota, USA) and with or without deep pericardial stich or heart positioner. Complete revascularization of the major coronary artery branches was completed in off-pump fashion, although graft selection and how to design the bypass were determined by each surgeon's preference. Distal anastomosis to the coronary artery with arterial graft was performed using single 8-0 polypropylene suture in continuous fashion while 7-0 polypropylene suture was used for anastomosis with vein grafts. As for proximal anasto $\neg$ mosis with the free vein grafts or radial artery to the aorta, 6-0 and 7-0 polypropylene sutures were used, respectively. The number of anastomoses were $3.3 \pm 0.9$. OPCAB with multi-arterial grafts was performed in 361 cases (42.4\%). Total arterial revascu $\neg$ larization was performed in 196 cases $(23.0 \%)$.

Follow up: Mean follow-up period of all patients was 25.7 months (range: 0.1-82.9 months). Postoperative follow-up coronary angiography was not routinely performed, due to

Table 2. Operative procedures, perioperative results in all cohort and the matched cohort

\begin{tabular}{|c|c|c|c|c|c|c|}
\hline Conversion to on-pump, n (\%) & $4(2.5)$ & $9(1.3)$ & 0.276 & $4(2.8)$ & $6(4.3)$ & 0.749 \\
\hline \multicolumn{7}{|l|}{ Postoperative complications, n (\%) } \\
\hline Wound infection & $1(0.6)$ & $6(0.9)$ & 0.902 & $1(0.7)$ & $1(0.7)$ & 1.000 \\
\hline New CVA & $1(0.6)$ & $7(1.0)$ & 0.911 & $1(0.7)$ & $3(2.1)$ & 0.615 \\
\hline New dialysis & $5(3.2)$ & $4(0.6)$ & $0.014 *$ & $3(2.1)$ & $1(0.7)$ & 0.622 \\
\hline In-hospital mortality, n (\%) & $5(3.2)$ & $8(1.2)$ & 0.075 & $4(2.8)$ & $5(3.6)$ & 0.811 \\
\hline Length of ICU stay (day), median IQR & $5(5-7)$ & $5(5-6)$ & 0.276 & $5(5-7)$ & $5(5-8)$ & 0.298 \\
\hline
\end{tabular}

$E F$, ejection fraction; CVA, cerebrovascular accident; ICU, intensive care unit; IQR, interquartile range

Table 3. Multivariable risk regression analysis of prognostic factors for in hospital death (all patient cohort and the matchedpropensity score cohort)

\begin{tabular}{|c|c|c|c|c|c|c|}
\hline Age & 1.04 & $0.97-1.12$ & 0.268 & 1.01 & $0.92-1.12$ & 0.772 \\
\hline Female vs. male & 2.23 & $0.75-6.65$ & 0.150 & 6.23 & $1.26-30.70$ & $0.025 *$ \\
\hline Renal insufficiency & 3.54 & $1.06-11.83$ & 0.040 & 3.96 & $1.06-14.74$ & 0.041 * \\
\hline Preoperative IABP & 2.62 & $0.73-9.42$ & 0.141 & 1.74 & $0.39-7.66$ & 0.467 \\
\hline
\end{tabular}

RR, risk ratio; $\mathrm{Cl}$, confidence interval; COPD, chronic obstructive pulmonary disease; NYHA, New York Heart Association; IABP, intra-aortic balloon pump 
Table 4. Prognostic factors for all-cause death in the long-term follow up (all patient cohort and the matched-propensity score cohort) analyzed by multivariable Cox's proportional hazard model

\begin{tabular}{|c|c|c|c|c|c|c|}
\hline Age & 0.99 & $0.96-1.02$ & 0.603 & 0.99 & $0.94-10.4$ & 0.654 \\
\hline Female vs. male & 1.09 & $0.65-1.85$ & 0.727 & 1.31 & $0.52-3.33$ & 0.568 \\
\hline NYHA & 1.61 & $1.02-2.54$ & 0.042 & 2.90 & $1.27-6.59$ & $0.011 *$ \\
\hline Recent myocardial infarction & 1.39 & $0.72-2.70$ & 0.332 & 0.63 & $0.18-2.21$ & 0.474 \\
\hline Renal insufficiency & 3.25 & $1.92-5.49$ & $<0.001$ & 5.53 & $2.19-13.99$ & $<0.001 *$ \\
\hline Preoperative IABP & 0.61 & $0.33-1.15$ & 0.129 & 0.39 & $0.15-1.02$ & 0.056 \\
\hline \multicolumn{7}{|l|}{ Diagnosis } \\
\hline
\end{tabular}

HR, hazard ratio; Cl, confidence interval; COPD, chronic obstructive pulmonary disease; NYHA, New York Heart Association; IABP, intra-aortic balloon pump

Table 5. Prognostic factors for postoperative MACCE (all patient cohort and the matched-propensity score cohort) analyzed by multivariable Cox's proportional hazard model

\begin{tabular}{|c|c|c|c|c|c|c|}
\hline Variables & $\mathrm{HR}$ & $95 \% \mathrm{Cl}$ & $P$-value & $\mathrm{HR}$ & $95 \% \mathrm{Cl}$ & $P$-value \\
\hline Age & 0.99 & $0.97-1.02$ & 0.821 & 0.98 & $0.95-1.02$ & 0.414 \\
\hline Female vs. male & 0.89 & $0.59-1.34$ & 0.575 & 1.31 & $0.66-2.60$ & 0.434 \\
\hline NYHA & 1.46 & $1.02-2.07$ & 0.036 & 2.26 & $1.23-4.14$ & $0.008^{*}$ \\
\hline Recent myocardial infarction & 1.58 & $0.96-2.62$ & 0.074 & 1.10 & $0.44-2.76$ & 0.834 \\
\hline Renal insufficiency & 2.45 & $1.62-3.71$ & $<0.001$ & 3.21 & $1.67-6.17$ & $<0.001 *$ \\
\hline Preoperative IABP & 0.91 & $0.56-1.45$ & 0.680 & 0.71 & $0.36-1.38$ & 0.307 \\
\hline \multicolumn{7}{|l|}{ Diagnosis } \\
\hline
\end{tabular}

MACCE, major adverse cardiac and cerebrovascular event; HR, hazard ratio; $\mathrm{Cl}$, confidence interval; COPD, chronic obstructive pulmonary disease; NYHA,

New York Heart Association; IABP, intra-aortic balloon pump

the unavailability of this modality with insurance reimbursement in Thailand, but was considered in patients who developed clinical symptoms suspecting possible cardiac ischemia.

Statistical analysis: Baseline characteristics, operative data, postoperative complications, and rates of outcomes were compared using Pearson Chi-square test (categorical variables), Student's t-test (continuous variables), and MannWhitney U test (continuous, skewed data). The KaplanMeier method with the attachment of the number of patients at risk was used for showing overall survival between groups. We analyzed using both the all-patient cohort and matchedpropensity score cohort. 
To reduce confounding, we used propensity scores to match the patients with Low EF to the patients with Faired EF. Logistic regression analysis was used to calculate a propensity score, which evaluates the unbalanced variables of patient characteristics. The variables included age, gender, BMI, NYHA, CCS, COPD, PVD, history of recent MI, preoperative renal insufficiency, and preoperative IABP use. The mean propensity score of these 2 groups was $0.30 \pm 0.18$ (Table 1). Matched propensity score was applied to balance the patient characteristics between the two groups. Propensity score matching resulted in the selection of 282 patients (Low EF group, $N=141$; Faired EF group, $N=141$, Table 1). Results from the all-patient cohort and matched propensity score analysis were evaluated.

The standardized differences (STD) between groups after matching was demonstrated. An STD of 0.2 or more indicates covariates were imbalanced between groups. The association between $\mathrm{EF}$ (EF less than 35 vs. $\mathrm{EF} \geqq 35$ or more (\%) and inhospital mortality was analyzed by multivariable risk regression analysis and demonstrated with risk ratio (RR) with $95 \%$ confidence interval (CI).

The association between EF and MACCE as well as allcause death in the long-term follow up were analyzed by multivariable Cox's proportional hazard model, demonstrated with hazard ratio (HR) with $95 \%$ confidence interval (CI). The significance level of the $P$-value was set at less than 0.05 . Statistical analyses were performed with STATA software version 16.0 (StataCorp, CS, TX, USA).

\section{RESULTS}

The comparison of operative procedures, perioperative results, and the outcome in the long-term follow up between these two groups are shown in Table 2. In the all cohort, the occurrence of postoperative new dialysis $(3.2$ vs. $0.6 \%, P=$ 0.014 ) was significantly different between these two groups. However, in the matched cohort, all variables, including major postoperative complications and in-hospital mortality, were not statistically different. (Table 2)

The multivariable risk regression analyses to identify prognostic factors for in-hospital mortality is shown in Table 3. In the matched cohort, gender, COPD, and preoperative renal insufficiency were detected as significant risk factors (gender, female; RR 6.23, $P=0.025$; COPD, RR 6.73, $P=$ 0.012 ; preoperative renal insufficiency, RR 3.96, $P=0.041$, Table 3), while Low EF was not a significant risk factor $(P=$ 0.398) for hospital death. (Table 3)

Table 4 shows the results of the analysis by multivariable Cox's proportional hazard model to identify prognostic factors for all-cause death in the follow-up periods both in the all cohort and matched cohort. In the matched cohort as well as in the all cohort, Low EF (HR 2.72, P = 0.029), COPD (HR 7.84, $P<0.001$ ), NYHA class (HR 2.9, $P=0.011$ ), and preoperative renal insufficiency (HR 5.53, $P<0.001$ ) were significant risk factors. (Table 4)

Table 5 shows the prognostic factors for postoperative MACCE in the follow-up periods both in the all cohort and

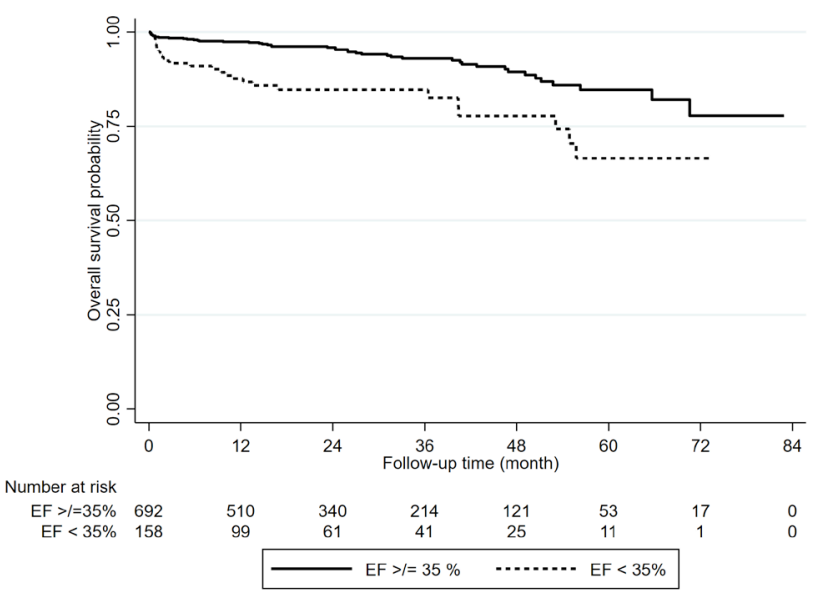

Figure 1A. Survival rate at 5 years in the Low EF or the Faired EF group in all cohorts.

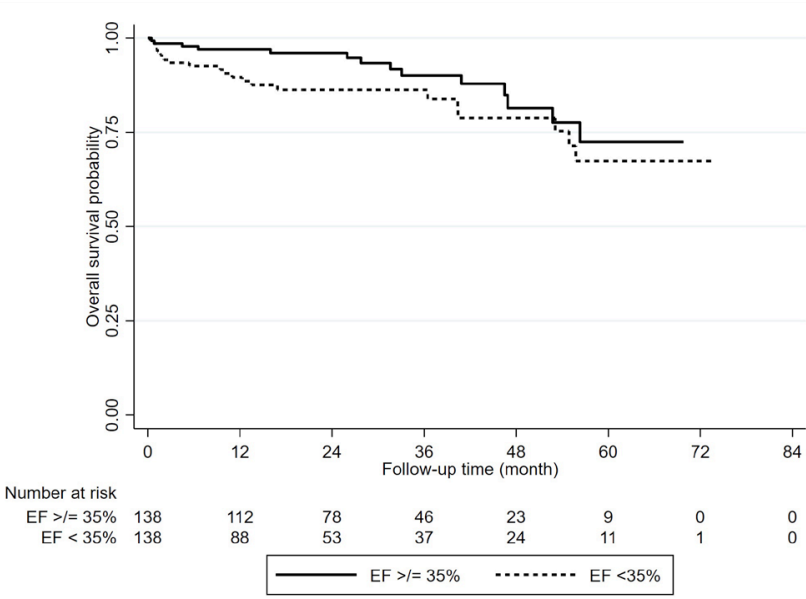

Figure 1B. Survival rate at 5 years in the Low EF or the Faired EF group after matched propensity score analysis.

matched cohort analyzed by multivariable Cox's proportional hazard model. In the matched cohort, COPD (RR 4.45, $P$ $<0.001)$, NYHA class $(\mathrm{RR}=2.26, \mathrm{P}=0.008)$, and preoperative renal insufficiency $(\mathrm{RR}=3.21, \mathrm{P}<0.001)$ were detected as significant risk factors for perioperative MACCE, while Low $\mathrm{EF}$ was not a significant risk factor both in the all cohort $(P$ $=0.098$, Table 5) and matched cohort $(P=0.111)$. (Table 5)

Figure $1 \mathrm{~A}$ and $1 \mathrm{~B}$ show overall survival rate between the Low EF and Faired EF groups in the all cohort (Figure 1A) and in the matched-propensity score cohort (Figure 1B). In the all cohort, the survival rate at 5 years in the Low EF or Faired EF groups were $67.4 \pm 4.1 \%$ and $86.1 \pm 2.9 \%$, respectively $(P<0.001$, Figure $1 \mathrm{~A})$. (Figure $1 \mathrm{~A})$ In the matched cohort, the survival rate at 5 years in the Low EF group was significantly inferior to that of the Faired EF group $(66.5 \pm 6.4 \%$ vs. $86.5 \pm 4.5 \%, P=0.008$, Figure 1B). (Figure 1B)

Figure $2 \mathrm{~A}$ and $2 \mathrm{~B}$ show freedom from MACCE rate 


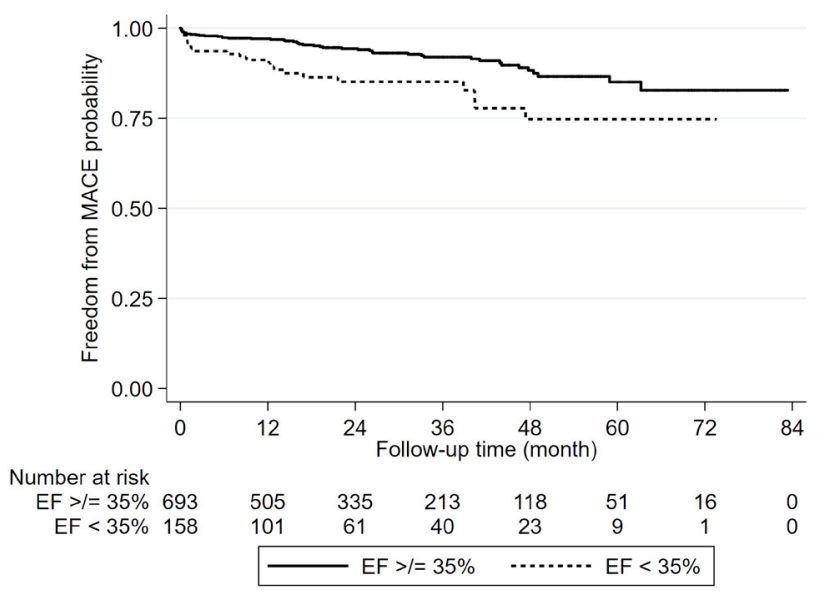

Figure 2A. Freedom from MACCE at 5 years in the Low EF or the Faired EF group in all cohorts.

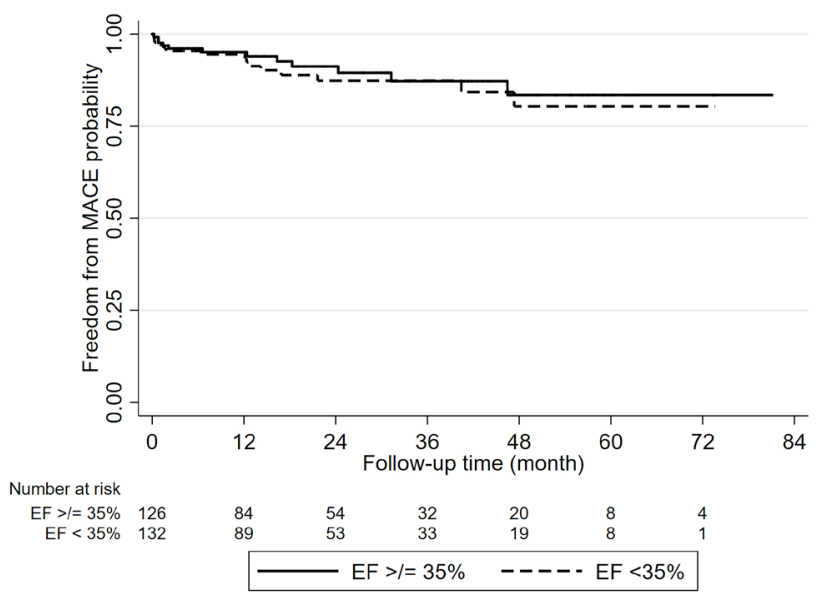

Figure 2B. Freedom from MACCE at 5 years in the Low EF or the Faired EF group after matched propensity score analysis.

between the Low EF and Faired EF groups in the all cohort (Figure 2A) and in the matched-propensity score cohort (Figure 2B). (Figure 2A) In the all cohort, freedom from MACCE at 5 years in these two groups were $63.3 \pm 5.2 \%$ and $74.2 \pm 3.7 \%$, respectively $(P=0.001$, Figure $2 \mathrm{~A})$. In matched cohort, the Low EF group was relatively inferior to that of the Faired EF group, although it was not statistically significant $(62.0 \pm 9.6 \%$ vs. $75.0 \pm 8.9 \%, P=0.078$, Figure $2 \mathrm{~B})$. (Figure $2 \mathrm{~B})$

\section{DISCUSSION}

There are a variety of postoperative complications in relation to the establishment of cardiopulmonary bypass (CPB), including stroke or cross-clamp induced aortic injury. However, OPCAB does not accompany these kinds of complications and can provide pulsatile circulation to every organ all through the operation. Therefore, the choice of this mode for high-risk patients who have several comorbidities, such as a history of cerebrovascular disease or LV dysfunction, would lead to a risk reduction [Christakis 1989; Kuroda 2016; Appoo 2004; Topkara 2005; Pieri 2016].

Previous reports emphasized that the operative mortality rate after $\mathrm{CABG}$ for patients with low EF were high [Pieri 2016; Jarral 2011], but several recent reports from Japan showed more favorable outcome has been achieved with the sophistication of the strategy of OPCAB even for this cohort (0.5-2.5\%) [Kuroda 2016; Fukui 2014; Ueki 2016]. Kuss et al. emphasized the superiority of OPCAB to on-pump CABG in hard endpoints with their review and meta-analysis of propensity score [Kuss 2010]. Similarly, Keeling et al., compared the outcomes of CABG for the patients with Low EF from the STS national database and concluded patients with LV dysfunction would preferentially benefit from OPCAB possibly because of the evolution or standardization of surgical techniques [Keeling 2013].

The central message from this study is that LV dysfunction itself is not a significant risk factor for mortality in the early postoperative period after OPCAB, however, it is surely a significant negative influencer for it in the midterm period. The details of preoperative patient clinical profiles imply that potentially low EF patients have multiple comorbidities than faired EF counterparts and such multi-preoperative factors may influence the hard endpoints in the midterm.

For example, postoperative renal insufficiency was a strong negative factor on overall death in the early period and MACCE in the midterm period assessed by multivariate analysis both in the all cohort and matched cohort. In general, renal dysfunction itself has been thought a risk factor for intraoperative and postoperative adverse events [Kuss 2010; Cooper 2006; Komiya 2009]. McCullough et al. proposed some explanations for the association between renal dysfunction and poor prognosis after coronary revascularization procedures as follows: (1) excess comorbidities in patients with chronic kidney disease; (2) excess toxicities in with chronic kidney disease; and (3) a special adverse pathobiological state in which there is acceleration of atherosclerotic, myocardial disease [Komiya 2009]. Considering that patients with preoperative renal dysfunction typically have multi-operative risk factors, OPCAB seems to be quite suitable for this cohort. This idea has been emphasized from several reports [Kuss 2010; McCullough 2002].

Complete revascularization with multiple arterial grafts for many target vessels is required especially for the patients with $\mathrm{LV}$ dysfunction brought by relatively broad ischemia from multiple impaired coronary flow [Fukui 2014; Puskas 2003; Carr 2002; Bouchart 2001; Kim 2001]. In complicated cases, OPCAB surgeons confront two hurdles: One is the technical demand to complete revascularization with multiple arterial grafts in beating fashion, which is more difficult than CABG under arrested heart [Taggart 2016]. Another hurdle is the risk of emergence of critical arrhythmia requiring pump conversion. Kuroda et al. recently reported the detailed information of patients who experienced the unplanned pump conversion, and major reasons were the elevation of pulmonary 
artery pressure and arrhythmia [Kuroda 2016]. Many reports show how important it is to use several mechanical devices to complete this maneuver safely for high-risk groups [Craver 2001; Vohra 2006]. In our unit, prophylactic IABP support for patients with $L V$ dysfunction or left main tract stenosis before or at the beginning of the operation routinely has been used, and it seems to have worked well from the outcome that in all cohort, pump conversion rate was $2.5 \%$ in the Low EF group and $1.3 \%$ in the Faired EF group, respectively $(P=$ 0.276 ), and in matched propensity score analysis, $2.8 \%$ and $4.3 \%$, respectively $(P=0.749)$.

This study has some limitations. The number of all patients in this study was small. Postoperative angiographic data could not be obtained in all patients. Furthermore, this was a retrospective observational study and was not randomized. Besides, this study did not compare the outcome of OPCAB and $\mathrm{ONCAB}$ in the same period because the number within the ONCAB group so far in our hospital has been quite small. This would make it difficult to do a statistical analysis properly between the two groups. So, the present study can't show the superiority of OPCAB to ONCAB. Moreover, the number of perioperative and postoperative clinical parameters in this study was not large.

In conclusion, OPCAB seems acceptable and beneficial for the patients with $L V$ dysfunction, referring to the fact that low EF is not a significant risk factor for hospital death both in the all and matched cohort. However, it is reality that it is a significant risk factor for overall death in the midterm period in both cohorts, although the multi-perioperative clinical factors also are influential.

\section{REFERENCES}

Appoo J, Norris C, Merali S, et al. 2004. Long-term outcome of isolated coronary artery bypass surgery in patients with severe left ventricular dysfunction. Circulation. 110: II13-7.

Bouchart F, Tabley A, Litzler PY, et al. 2001. Myocardial revascularization in patients with severe ischemic left ventricular dysfunction. Long term follow-up in 141 patients. Eur J Cardiothorac Surg. 20: 1157-62.

Carr JA, Haithcock BE, Paone G, et al. 2002. Long-term outcome after coronary artery bypass grafting in patients with severe left ventricular dysfunction. Ann Thorac Surg. 74: 1531-6.

Christakis GT, Ivanov J, Weisel RD, Birnbaum PL, David TE, Salerno TA. 1989. The Cardiovascular Surgeons of the University of Toronto: The changing pattern of coronary artery bypass surgery. Circulation. 80 Suppl: I151-I161.

Cooper WA, Brien SM, Thourani VH, et al. 2006. Impact of renal dysfunction on outcomes of coronary artery bypass surgery. Results from the Society of Thoracic Surgeons National Adult Cardiac Database. Circulation. 113:1063-1070.

Craver JM, Murrah CP. 2001. Elective intraaortic balloon counterpulsation for high-risk off-pump coronary artery bypass operations. Ann Thorac Surg. 71: 1220-1223.

Fukui T, Tabata M, Takanashi S. 2014. Long-term outcomes after offpump coronary artery bypass grafting in left ventricular dysfunction. Ann
Thorac Cardiovasc Surg. 20: 143-149.

Jarral OA, Srdjan Saso, Athanasiou T. 2011. Off-pump coronary artery bypass in patients with left ventricular dysfunction: A meta-analysis. Ann Thorac Surg. 92:1686-94.

Keeling WB, Williams ML, Slaughter MS, Zhao Y, Puskas JD. 2013. Off-pump and on-pump coronary revascularization in patients with low ejection fraction: a report from the Society of Thoracic Surgeons National Database. Ann Thorac Surg. 96:83-8.

Kim KB, Lim C, Ahn H, Yang JK. 2001. Intraaortic balloon pump therapy facilitates posterior vessel off-pump coronary artery bypass grafting in high-risk patients. Ann Thorac Surg. 71: 1964-1968.

Komiya T. 2009. Coronary artery bypass surgery for patients with renal failure. J Jpn Coron Assoc. 15: 246-251.

Kuroda K, Kato ST, Kuwaki K, et al. 2016. Early postoperative outcome of off-pump coronary artery bypass grafting: A Report from the highestvolume center in Japan. Ann Thorac Cardiovasc Surg. 22: 98-107.

Kuss O, von Salviati B, Börgermann J. 2010. Off-pump versus on-pump coronary artery bypass grafting: a systematic review and meta-analysis of propensity score analyses. J Thorac Cardiovasc Surg. 140: 829-35.

Marui A, Okabayashi H, Komiya T, et al. 2012. Benefits of off-pump coronary artery bypass grafting in high-risk patients. Circulation. 126 : $151-7$.

Matsuo S, Imai E, Horio M, et al. 2009. Revised equation for estimated GFR serum creatinine in Japan. Am J Kidney Dis. 53:982-92.

McCullough PA. 2002. Cardiorenal risk: an important clinical intersection. Rev Cardiovasc Med. 3:71-76.

Møller CH, Penninga L, Wetterslev J, Steinbrüchel DA, Gluud C. 2008. Clinical outcomes in randomized trials of off- vs. on-pump coronary artery bypass surgery: systematic review with meta-analyses and trial sequential analyses. Eur Heart J. 29:2601-16.

Pieri M, Belletti A, Monaco F, et al. 2016. Outcome of cardiac surgery in patients with low preoperative ejection fraction. BMC Anesthesiology. 16:97.

Puskas JD, Williams WH, Duke PG, et al. 2003. Off-pump coronary artery bypass grafting provides complete revascularization with reduced myocardial injury, transfusion requirements, and length of stay: a prospective randomized comparison of two hundred unselected patients undergoing off-pump versus conventional coronary artery bypass grafting. J Thorac Cradiovasc Surg. 125: 797-808.

Shroyer AL, Grover FL, Hattler B, et al. 2009. On-pump versus offpump coronary-artery bypass surgery. N Engl J Med. 361:1827-37.

Taggart DP. 2016. Off-pump coronary artery bypass grafting (OPCABG)—a 'personal' European perspective. J Thorac Dis. 8 (Suppl 10):S829-S831.

Topkara VK, Cheema FH, Kesavaramanujam S, et al. 2005. Coronary artery bypass grafting in patients with low ejection fraction. Circulation. 112: I344-50.

Ueki C, Miyata H, Motomura N, Sakaguchi G, Akimoto T, and Takamoto S. 2016. Off-pump versus on pump coronary artery bypass grafting in patients with left ventricular dysfunction. J Thorac Cardiovasc Surg. 151:1092-8.

Vohra HA, Wadih R, Dimitri WR. 2006. Elective Intraaortic balloon counterpulsation in high-risk off-pump coronary artery bypass grafting. J Card Surg. 21:1-5. 